\title{
The Evolutionary Theories of Aging Revisited - A Mini-Review
}

\author{
Predrag Ljubuncic Abraham Z. Reznick \\ Department of Anatomy and Cell Biology, Rappaport Faculty of Medicine, Technion - Israel Institute of Technology, \\ Haifa, Israel
}

\author{
Key Words \\ Evolution · Aging $\cdot$ Longevity $\cdot$ Genomic studies $\cdot$ Nuclear \\ factor kappa B
}

\begin{abstract}
This short review portrays the evolutionary theories of aging in the light of the existing discoveries from genomic and molecular genetic studies on aging and longevity. At the outset, an historical background for the development of the evolutionary theories of aging is presented through the works of August Weismann (programmed death and the germ plasm theories) including his exceptional theoretical postulation, later experimentally validated by the existence of cell division limits. Afterwards, the theory of mutation accumulation of Peter Medawar and the theory modification by Charlesworth (late-life mortality plateau) are presented as well as the antagonistic pleiotropy hypothesis of George Williams, and the disposable soma theory of Kirkwood and Holliday. These theories are discussed in the light of the different research studies, which include studies on insulin signaling and longevity, the possibility that nuclear factor kappa B may be a major mediator of aging, studies of anti-aging Sirtuins and studies on heat shock proteins and longevity and on gene sets as biomarkers of aging. Finally, the proposals for future research in biogerontology, such as studies on the control of protein synthesis, validation of biomarkers of aging, understanding the biochemistry of longevity and research in the field of gerontologic pathology are presented.
\end{abstract}

Likewise, further attention is suggested regarding the work on telomere shortening, stem cells and studies on understanding the biochemical and molecular basis for longevity in centenarians.

Copyright $\odot 2009$ S. Karger AG, Basel

\section{Introduction}

\section{Relation between Aging and Darwin's Evolutionary Theory}

Darwin's evolutionary theory is based on the concept that random and heritable variation of biological traits between individuals (caused by mutations) will lead to natural selection for preferential reproduction of those individuals who are particularly fit in a given environment. On the other hand, the process of aging increases vulnerability and ultimately leads to the death of organisms. In view of that, evolutionary theories of aging are those theories that try to explain the developmental program formed by biological evolution and the evolution of aging through interplay between the processes of mutation and selection. They also had an influence on thinking about the process of aging patterns, thus forming a picture that may explain how longevity is distributed across the species. Finally, these theories enable us to make theoretical predictions which can be tested in experimental laboratory and field conditions.

\section{KARGER \\ Fax +4161306 1234 \\ E-Mail karger@karger.ch}

www.karger.com
(C) 2009 S. Karger AG, Basel

0304-324X/09/0552-0205\$26.00/0

Accessible online at:

www.karger.com/ger
A.Z. Reznick

Department of Anatomy and Cell Biology, Faculty of Medicine

Technion - Israel Institute of Technology

Haifa 31096 (Israel)

Tel. +972 4829 5388, Fax +972 4829 5403, E-Mail reznick@tx.technion.ac.il 


\section{Historical Account}

\section{How the Evolutionary Theories of Aging Have}

Evolved

Before the theory of evolution, the process of aging was conceived in the same way that moving parts wear out, machines break down and all things deteriorate slowly over time. After the development of the theory of evolution, scientists began to wonder why evolution had produced such complex and well-adapted creatures that were so successful at surviving from conception through to adulthood, but which then fell into decay and died.

The problem of how aging evolved was first debated by August Weismann [1] who developed the theory of programmed death which proposed that aging evolved to the advantage of the species, not the individual, and that there must be an evolutionary advantage to having only a limited lifespan. The evolutionary argument was used to suggest that older members of a species are expected to die of old age by specific death mechanism of natural selection meant to eliminate old members of population so that they would no longer compete with younger generations for food and other resources. Accordingly, one of the most important concepts provided by Weismann was the necessity of reproduction in a world that invariably causes degradation of the individual [2].

On the other hand, there is compelling evidence against the theory of programmed death, such as high levels of extrinsic mortality observed by Medawar (his work to be discussed in greater detail later). For example, animals living in harsh conditions in their natural habitat survive for only relatively short period of time compared to those living in the protected conditions of captivity, which is opposite to what would be expected from the programmed death theory. For instance, 9 out of 10 mice living in the wild will die before the age of 10 months, whereas the same mice raised in captivity have an average lifespan of 24 months. Also, the median lifespan of chimpanzees (Pan troglodytes) living in captivity is between 23 years (males) and 30 years (females), and almost $20 \%$ of captive female chimpanzees survive to age $50[3,4]$. In addition to many advantageous environmental factors, appropriate medical treatment (including vaccinations) may also be an important life-prolonging factor under these circumstances. On the other hand, in wild conditions, the median lifespan of chimpanzees is only 8 years and almost none survive to age 50 [4]. Austad [5] compared the survival and reproduction of 2 groups of closely related opossums; 1 of those has lived on an isolated island, disconnected from the mainland for 4,500 years, and which has been subject to diminished levels of predation pressure and thus less selection for extremely rapid reproduction. According to the measurement of longevity as well as the collagen biochemistry, Austad has found that the island population aged more slowly in comparison to the mainland population [5]. Also, the longevity of birds, turtles and porcupines reflects their relative invulnerability to predation. Birds, which live 5-10 times longer than comparably sized mammals, have apparently had enough time available to evolve protective mechanisms, such as antioxidant pathways and controls of oncogene expression, that may account for their resistance to age-related pathology. Accordingly, these studies prove that different selective pressures on genes alter rates of growth and development.

Observations like these are common for many biological species. In reality, very few individuals of any species living in the wild survive long enough for there to be any evolutionary pressure to set an age limit.

\section{Importance of Weismann's Work}

In order to explain the biological mechanism of his theory of programmed death, Weismann came to an idea that there is a specific limitation on the number of divisions that somatic cells might undergo in the course of an individual life and that this number, like the life span of individual generations of cells, is already determined in the embryonic cell [6]. By this means, the different life span of animals would be dependent on the number of cell generations in which there is a specific norm for each different species [6]. This exceptional theoretical speculation over the existence of a cell division limit was experimentally confirmed in 1961 by Hayflick and Moorhead [7] who found that normal human embryo fibroblasts undergo a finite number of cell divisions, and then they 'age' and 'die'. In the case of the fibroblasts the limit was about 50 cell divisions. These results have been confirmed in many laboratories for a variety of cell types and became known as the Hayflick limit [8]. It should be noted here that the process of apoptosis (programmed cell death), which was distinguished from traumatic cell death by John Foxton Ross Kerr [9], or autophagy pathway for cellular death [10] is irrelevant to the above discussions focusing on the death-mechanism of the whole organism and not to some of its specific somatic cells.

To conclude, Weismann contributed the first evolutionary theory of aging which drew the attention of other researchers. Also, on the basis of theoretical inspiration alone, he rightly predicted the existence of a cell division 
limit. Finally, he abandoned his first concept and suggested afterward that aging evolved because organisms that segregate germ and soma must invest additional resources to reproduce instead of maintaining the soma, and this renunciation of the soma results in aging. Thus, he formulated the germ plasm theory, i.e. that the body is divided into the germ cells (sperm or ova cells), which transmit hereditary information to the offspring and to all other (somatic) cells [11]. It should be emphasized that the disposable soma theory of Thomas Kirkwood, which states that organisms must reach a balance between the resources they invest in soma maintenance and resources invested in reproduction [12], is based to a certain extent on Weismann's ideas.

\section{Contemporary Evolutionary Theories of Aging}

Basically, the evolutionary theory of aging proposes effectively 2 models for how aging can evolve: (1) the theory of mutation accumulation and (2) the antagonistic pleiotropy hypothesis. The theory of mutation accumulation originates from Peter Medawar's ideas in which he argued that diminishing selection would lead to the accumulation of late-acting harmful genes. In George Williams's model of antagonistic pleiotropy, aging evolves due to the pleiotropic effect of genes that are beneficial early in life and then harmful at late ages.

\section{The Mutation Accumulation Theory Suggested by Medawar}

The basic observations that the force of natural selection declines with age were developed by Peter Medawar [13]. Since all organisms eventually die from different causes (e.g. diseases, accidents, predation), genes beneficial early in life are favored by natural selection over genes beneficial late in life [13]. Consequently, the greatest contribution to create a new generation comes from young, not from old organisms. Thus, the power of natural selection fades with age, making it possible for hazardous lateacting genes to exist [14].

The mutation accumulation theory of aging of Medawar considers aging as a byproduct of natural selection. According to this theory, aging has no adaptive traits because natural selection does not occur in longlived animals and provides little additional contribution to offspring numbers. In other words, old age is not under selective pressure per se, and there is no evolutionary mechanism to rid a population of mutations that cause detrimental effects only in old animals [13].

Evolutionary Theories of Aging
The probability of an individual reproducing is age dependent. It is zero at birth and reaches a peak in young adults. Then it decreases due to the increased probability of death linked to various external (predators, illnesses, accidents) and internal (senescence) causes. Under such conditions, deleterious mutations expressed at a young age are severely selected against because of their highly negative impact on fitness (number of offspring produced). On the other hand, deleterious mutations expressed later in life are relatively neutral to selection because their bearers have already transmitted their genes to the next generation. Mutation accumulation theory also predicts that the dependence of progeny lifespan on parental lifespan should not be linear. Instead, this dependence should have nonlinear shape with an increasing slope for the dependence of progeny lifespan on parental lifespan for longer-lived parents. This prediction follows directly from the original statement of the mutation accumulation theory that the equilibrium gene frequency for deleterious mutations should increase with age at onset of mutation action because of weaker (postponed) selection against later-acting mutations [15]. Thus, it was argued, if the ages at death were indeed determined by accumulated late-acting deleterious mutations, one would expect this slope to become steeper with higher parental ages at death [16]. Indeed, the analysis of the reliable and accurate genealogical data on familial longevity in European royal and noble families found that the regression slope for the dependence of offspring lifespan on parental lifespan increases with parental lifespan, as predicted by the mutation accumulation theory [16].

According to Strehler's criteria for the aging process, it is cumulative, universal, progressive, intrinsic and detrimental, which means it is omnipresent [17]. In addition, according to the predictions of the mutation accumulation theory, mechanisms that limit lifespan may be placed into 3 categories: all aging mechanisms that involve a gradual deterioration of cellular and metabolic processes with age through the appearance of cellular and molecular damage late in the life (oxidative damage, somatic DNA mutation, telomere shortening, etc.); other mechanisms that are linked to young adulthood (cell senescence, caloric restriction and insulin-signaling pathway which regulates caloric restriction), and external mechanisms which are unavoidable consequences of old age such as inflammation response, caused by infection and pathogenic invasion in old age [18]. While categories 2 and 3 might be similar among species, the first category is different between various species. 


\section{Late-Life Mortality Plateau: Charlesworth's}

Modified Mutation Accumulation Theory

Under traditional evolutionary models mortality rates should increase monotonically after sexual maturity. However, the equilibrium frequencies of deleterious alleles affecting late life are lower than predicted under traditional models. The discovery of late-life mortality plateaus $[19,20]$, in which cohort mortality rates appear to plateau or even decrease at late ages, challenged the validity of evolutionary explanations of aging. In that way, Charlesworth [21] presented a modified mutation accumulation model in which mortality plateaus are predicted to occur at late ages if alleles affecting fitness do so for more than 1 specific age range class (moderate age specificity). Under the modified mutation accumulation model, late-life mortality plateaus were predicted whether deleterious alleles affected fitness during a 'window' of just a few age classes or affected fitness cumulatively at all ages after a given age. Computer simulations testing this model of age specificity were consistent with existing data on late-life mortality [21]. Recently, Reynolds et al. [22] evaluated the mutation accumulation model by testing the degree of age specificity of segregating alleles affecting fitness in Drosophila melanogaster. They assessed age specificity by measuring the homozygous fitness effects of segregating alleles across the adult life span and then calculated genetic correlations of these effects across age classes. The authors found for both males and females that allelic effects were age specific with effects extending over 1-2 weeks across all age classes, which was consistent with modified mutation accumulation theory [22]. Thus, these results point out that Charlesworth's modified mutation accumulation theory can both explain the origin of senescence and predict late-life mortality plateaus.

\section{Antagonistic Pleiotropy Model Suggested by Williams}

The antagonistic pleiotropy model by Williams ('pay later' theory) proposes that some genes are beneficial at earlier ages but harmful at later ages. The genes with agerelated opposite effects are called pleiotropic genes [23]. Accordingly, the theory of antagonistic pleiotropy is based on 2 assumptions. First, it is assumed that a particular gene may have an effect not only on 1 feature but on several traits of an organism (pleiotropy). The second assumption is that these pleiotropic effects may affect individual fitness in antagonistic ways. For example, a gene that increases survival to reproductive age or reproductive output will be favored by natural selection if it decreases the chances of dying at age 10 or 20 . Thus, harmful late-acting genes can remain in a population if they have a beneficial effect early in life such as increasing fitness at early ages or increasing reproductive success. However, it was argued by Williams that natural selection will frequently maximize vigor in youth at the expense of vigor later on and thereby produce a declining vigor (aging) during adult life [23]. One frequently quoted example is that of Zahavi about the costly sexual ornaments of male birds that are crucial to attracting females, and thus vital to passing on genes to the next generation. However, these ornaments can be considered handicaps, e.g. the extravagant feathers of male peacocks limit the bird's movements, which can deter their ability to escape predators [24]. Therefore, the stipulation of the existence of pleiotropic antagonistic genes could explain the aging process. Such genes will be maintained in the population due to their positive effect on reproduction at young ages, despite their negative effects at old, postreproductive age by having negative effects that look exactly like the aging process. These initially verbal arguments were later proved mathematically by Charlesworth [15].

An example of antagonistic pleiotropy refers to replicative cellular senescence (cell division limit), which is known to suppress tumorigenesis by arresting cell growth [25]. This process, which suppresses tumorigenesis early in life, may promote cancer in later life because senescent cells stimulate other premalignant and malignant cells to proliferate and to form tumors $[26,25]$. Thus, there is a trade-off between the earlier protective effect of growth arrest because of cellular senescence and the later detrimental effect caused by cancer promotion.

The antagonistic pleiotropy theory may also explain the so called idea of reproductive cost or, more generally, of trade-offs between different traits of the organism in which reproduction may come with a cost for species longevity. The mutations favoring more intensive reproduction (more offspring produced) will be propagated in future generations even if these mutations have some deleterious effects in later life. For example, mutations causing overproduction of sex hormones, which may increase the sex drive, libido, reproductive efforts and success, may be favored by selection despite causing sex-related cancers (prostate and ovarian cancer) later in life.

The trade-offs between reproduction and longevity was also predicted by Williams, who was arguing that reproductive maturation is the most important landmark in the life-cycle for the evolution of senescence and that senescence may already theoretically begin right after re- 
productive maturation stage in development is reached. This prediction was tested in the fruit fly D. melanogaster and, contrary to this prediction, no linear relationship was found between the speed of development and longevity [27]. Also, the search for the trade-offs between longevity and reproduction was made in experiments with the nematode Caenorhabditis elegans where a number of long-lived mutants have been identified. When long-lived mutants were put under standard culture conditions together with normal wild-types individuals, neither of them exhibited a competitive advantage, contrary to theoretical evolutionary predictions $[28,29]$. However, when nematode cultures were alternatively fed and starved, mimicking cyclic field conditions in nature, the wildtype worms outcompeted (outnumbered) the long-lived mutant [29]. These findings, demonstrating that increasing lifespan may exhibit some fitness cost only in harsh conditions, provided limited support for the antagonistic pleiotropy theory of aging.

Also, pleiotropic antagonism forms the basis for the development of many age-related diseases. The examples for the mechanism of pleiotropic antagonism include amongst other, atherosclerosis, benign and malignant prostate hypertrophy, Alzheimer's disease and the reciprocal relationship between cellular senescence and cancer [for more details see 30]. Also, it is worth mentioning 'paradoxical antagonistic pleiotropy' which refers to the setting of bad alleles with deleterious effect early in life but which have beneficial late effects [31]. One quoted example is the allele at the plasminogen activator inibitor-1 locus which is found augmented in centenarians although, paradoxically, it is a predictor of recurrent myocardial infarctions in young people [32]. Thus, paradoxical antagonistic pleiotropy stands in contradiction to the 'pay later' proposal that genes are beneficial only at earlier ages but harmful at later ages.

\section{Antagonistic Pleiotropy Theory versus Mutation Accumulation Theory}

What are the main differences between these 2 evolutionary theories of aging? In short, the main difference between the 2 theories is that in the mutation accumulation theory, genes with negative effects at old age accumulate passively from one generation to the next, while in the antagonistic pleiotropy theory these genes are actively kept in the gene pool by selection [33]. However, it should be noted that both theories are widely accepted as applicable. Also, they are not mutually exclusive and both evolutionary mechanisms may operate at the same time [34].

Evolutionary Theories of Aging
The Disposable Soma Theory by Kirkwood and

Holliday

There were attempts to find a better name for the antagonistic pleiotropy theory and to specify in more detail how one and the same gene could have both deleterious and beneficial effects. The disposable soma theory, which was proposed by Kirkwood and Holliday [35], predicts that aging occurs due to the accumulation of damage during life and that failures of defensive or repair mechanisms contribute to aging $[12,35,36]$. It postulated a special class of gene mutations with antagonistic pleiotropic effects in which hypothetical mutations save energy for reproduction (positive effect) by partially disabling molecular proofreading and other accuracy promoting devices in somatic cells (negative effect). In other words, given finite resources, the more an animal expends on bodily maintenance, the less it can expend on reproduction, and vice versa. The distinction between somatic and reproductive tissues is therefore important because the reproductive cell lineage, or germ line, must be maintained at a level that preserves viability across the generations, whereas the soma needs only to support the survival of a single generation. Thus, the key feature of the disposable soma theory is its emphasis on the optimal balance between somatic maintenance and repair versus reproduction. The theory also suggests that multiple kinds of damage will accumulate in parallel within cells, as the same logic limits the investment in each of wide range of maintenance and repair functions. To conclude, the disposable soma theory closes the gap between mechanistic and evolutionary theories of aging by suggesting that aging results from progressive accumulation of molecular and cellular damage, as a direct consequence of evolved limitations in the genetic settings of maintenance and repair functions $[12,36]$.

\section{Evolutionary Theory of Aging and Research on Aging - Contradictions Exist}

One of the most intriguing phenotypes in the biology of aging is that there are animals that appear not to age. In fact, these animals (e.g. female turtles) may increase both survivorship and reproductive output with age [37]. These observations, which suggest some species may not age, are contradictory to classical evolutionary models of aging which predict that all species eventually age [38]. In addition, evolutionary theory was interpreted by evolutionary biologists in a way that the search for singlegene mutations or life-extending interventions with 
large positive effects on lifespan was considered a completely futile task. However, the discovery of different single-gene mutants with profoundly extended longevity was ultimately made. For example, a single-gene mutation (daf-2) more than doubles the lifespan of nematodes, keeping them active, fully fertile (contrary to predictions of the disposable soma theory) and having normal metabolic rates [39].

Rapid progress in human genomics raises the prospect of significantly increasing our knowledge of the determinants of human longevity. In the extensive search conducted over the last 3 decades for genes that affect aging in model organisms like yeast (Saccharomyces cerevisiae), nematode worms (C. elegans), and fruit flies (D. melanogaster), many genes have been identified that alter the rate of aging, as measured both by lifespan and Gompertzian increase in age-related mortality, but none has been found that abolishes the aging process altogether [40]. Aging should not be viewed as programmed, but instead as the result of the accumulation of somatic damage, owing to limited investments in maintenance and repair.

\section{Molecular Genetic Studies of Aging and Longevity}

\section{Insulin Signaling and Longevity}

In most cases, the genes with major effects on longevity have been found to regulate central aspects of metabolism, particularly with respect to organism energetic balance, such as insulin/insulin-like growth factor-1 signaling [41]. The studies of caloric restriction, mutant worms and flies as well as long lived mutant mice led to recognition of the centrality of insulin-like signaling in regulation of longevity in widely separated species of animals. In that way, the daf-2 signaling pathway is part of an endocrine system that regulates longevity, metabolism and development in C. elegans and is homologous to mammalian insulin and insulin-like growth factor-1 signaling pathway [42]. Daf-2 encodes the worm homolog of the insulin/insulin-like growth factor-1 receptor. Signaling from DAF-2 is mediated through the AGE-1 phosphatidylinositol 3-kinase, PDK-1, and AKT-1/2 kinases to antagonize DAF-16, orthologous to human FOXO, a forkhead transcription factor. In C. elegans, a conserved insulin-like signaling pathway promotes wild type lifespan, stress resistance and reproductive development [43]. Adult lifespan and stress resistance were found to be increased by mutations in the DAF-2/insulin receptorlike protein, AGE-1 (a homolog of the p110 catalytic subunit of phosphoinositide 3-kinase) or in AKT-1, PDK-1 or
SGK-1, 3 downstream targets of DAF-2 [39, 44, 45]. The major target of insulin-like signaling is the FOXO transcription factor, DAF-16, whose mammalian orthologs are FOXO1, FOXO3a and FOXO4 [46].

Recently, Selman et al. [47] offered evidence for lifespan extension and delayed age-related biomarkers in insulin receptor substrate (IRS) 1 null mice. The authors measured the life span of mice lacking either IRS 1 or 2, the major intracellular effectors of the insulin/insulinlike grow th factor-1 signaling receptors. Their provisional results indicate that female $\operatorname{Irs} 1^{-/-}$mice are long-lived. Furthermore, they displayed resistance to a range of agesensitive markers of aging including skin, bone, immune and motor dysfunction. In contrast, Irs $2^{-/-}$mice were short-lived, whereas $I r s 1^{+/-}$and Irs $2^{+/-}$mice of both sexes showed normal life spans. Thus, IRS1 signaling, an evolutionarily conserved pathway regulating mammalian lifespan, may be a point of intervention for therapies with the potential to delay age-related processes.

\section{Is Nuclear Factor Kappa B a Signaling Mediator of Aging?}

Genetic studies in model organisms such as yeast, worms, flies and mice that have lead to lifespan extension suggest that longevity is subject to regulation. In addition, various system-wide interventions in old animals can reverse features of aging. To better understand these processes, much effort has been put into the study of aging on a molecular level. In particular, genome-wide microarray analysis of differently aged individual organisms or tissues has been used to track the global expression changes that occur during normal aging. Although these studies consistently implicate specific pathways in aging processes, there is little conservation between the individual genes that change. To circumvent this problem, Adler et al. [48, 49] have recently developed a novel computational approach to discover transcription factors that may be responsible for driving global expression changes with age. They developed a systematic approach to identify combinatorial cis-regulatory motifs that drive age-dependent gene expression across different tissues and organisms. Integrated analysis of 365 micro arrays spanning 9 tissue types predicted 14 motifs as major regulators of age-dependent gene expression in humans and mice. The motif most strongly associated with aging was that of the transcription factor nuclear factor kappa $\mathrm{B}$ $(\mathrm{NF}-\kappa \mathrm{B})$. Accordingly, the transcription factor NF- $\mathrm{B}$ has been identified as a candidate activator of aging-related transcriptional changes in multiple human and mouse tissues. Inducible genetic blockade of NF- $\mathrm{B}$ for 2 
weeks in the epidermis of chronologically aged mice by adenovirus-mediated expression of dominantly active I $\mathrm{B} \alpha(\mathrm{Ad}-\mathrm{I} \kappa \mathrm{B} \alpha)$ reverted the tissue characteristics and global gene expression programs to those of young mice [48]. Also, age-specific NF- $\mathrm{B}$ blockade and orthogonal cell cycle interventions revealed that NF- $\kappa \mathrm{B}$ controls cell cycle exit and gene expression signature of aging in parallel, but not sequential pathways. These results identify a conserved network of regulatory pathways underlying mammalian aging and show that NF- $\mathrm{KB}$ is continually required to enforce many features of aging in a tissuespecific manner. Thus, genetic blockade of NF- $\kappa \mathrm{B}$ in the skin of chronologically aged mice $(\Delta$ SP-p50-ER transgenic mice) reversed the global gene expression program and tissue characteristics to those of young mice, demonstrating for the first time that disruption of a single gene is sufficient to reverse features of aging, at least for the short-term [48]. Age-associated genes whose expression was inhibited by NF- $\kappa \mathrm{B}$ blockade included those related to chromatin/transcriptional regulation (RAD50, $S M C 2 L 1, S M C 6 L 1$ and ATRX), protein modification/signal transduction (STK25, RAMP2 and HIP2), cell cycle/ growth control (DNAJC2 and IGFBP5) and mitochondria (ALAS2, GSTK1, and PTE1).

It should also be noted that experiments have shown that activation of NF-кB can induce muscle atrophy [50], insulin resistance [51] and neurotoxicity in Alzheimer's disease [52], 3 prevalent age-associated morbidities. Our work in vitro has shown that NF- $\mathrm{BB}$ activity increases in cultured muscle cells exposed to pro-inflammatory reactive nitrogen species, which are increasingly generated during aging, and that peroxynitrite may induce alternative activation of the NF- $\kappa$ B classic pathway of activation [53]. In addition, in our in vivo work we have observed a distinctive biphasic pattern of NF- $\kappa \mathrm{B}$ activation in atrophic muscles of old rats $[54,55]$. Interestingly, the human NFKB1 gene maps within a genetic locus on chromosome 4 that has been repeatedly associated with human longevity [56].

\section{Sirtuins-Anti-Aging Proteins}

Experiments in model organisms including yeast, worms, flies and mice reveal that several lifespan-related genes act through similar evolutionarily conserved pathways, of which the best characterized is the insulin/insulin-like growth factor 1 endocrine pathway. Among the genes that have been shown to regulate aging in different species are the SIR2 family and its functional orthologs that make up a family of deacetylase enzymes termed Sirtuins. Histone and protein deacetylases fall into 4 classes with the yeast proteins Rpd3 (class I), Hda1 (class II) and Sir2 (class III) serving as charter members of the 3 major classes [57].

Class III deacetylases, the regulatory Sirtuins, which are considered anti-aging proteins related to the yeast SIR2 and its mammalian ortholog SIRT1, are unique in that they require nicotinamide adenine dinucleotide (NAD) coenzyme as a cofactor which is involved with many types of oxidation reactions. As such, Sirtuin activity may be controlled by cellular [NAD]/[NADH] ratios and respond to changes in cellular metabolism [58]. Increased Sir2 activity has been reported to enhance the lifespan of C. elegans and D. melanogaster $[59,60]$.

\section{Heat Shock Proteins and Longevity}

The analysis of long lived mutant worms led to important findings. For example, it was observed that the lifespan extending mutations have in common an increased resistance to cellular damage caused by ultraviolet light, oxidizing agents and overheating. From that, it can be assumed that the increased resistance to such types of injuries is the link which may lead to longevity. In that way, heat shock genes may be candidates for such links because they become induced by a similarly wide range of stresses not only in worm and fly cells but also in human cells. Stress proteins, including the heat shock proteins (HSPs), function in combating the stress-induced damage. Experiments have shown that transgenic worms which are genetically engineered to have certain types of HSP upregulated, such as Hsp16 or Hsp70F, live much longer $[61,62]$. Moreover, transcriptional activator heat shock factor HSF-1, which regulates the heat-shock response, also influences aging in C. elegans. Hsu et al. [63] have shown that hsf-1 overexpression extends lifespan. They found that HSF-1, like the transcription factor DAF-16, is required for daf-2-insulin/ IGF-1 receptor mutations to extend life-span. Accordingly, they suggested that this is because HSF-1 and DAF-16 together activate expression of genes encoding small HSPs, which in turn promote longevity [63]. Thus, the role of multiple transcription factors in extending lifespan in yeast and worms raises the possibility that many transcriptional regulators can contribute to longevity [64].

\section{Mitochondrial Electron Transport Chain Gene Set - A Biomarker of Aging?}

Most age-related expression changes are specific for a given species, but genes in the electron transport chain pathway, show common age regulation in species from 
worms to humans. It was found that the electron transport chain gene set was similarly age-regulated in worms, flies, mice and humans by showing about a 2 -fold decrease in the overall level of expression in old age for all 4 species [65]. Zahn et al. found that genes involved in the electron transport chain show common age regulation in all 4 species, indicating that these genes may be exceptionally good markers of aging [65].

Most of the electron transport chain genes are located in the nuclear DNA and encode components of mitochondrial enzyme complexes that are the primary source of generation of free radicals in the cell. Oxidative damage from the mitochondrial free radicals may accumulate with time and thereby decrease overall cell function and ultimately limit an organism's lifespan [66]. The lifespans of worms and humans differ by approximately 2,000-fold ( 2 weeks vs. 80 years), and the slope of age-related changes in expression for this pathway scales with lifespan such that old worms and old humans showed a similar overall decrease in expression levels. Although indicating that genes may be exceptionally good markers of aging, Zahn et al. [67] saw no overall correlation of age regulation between mice and humans, thus suggesting that aging processes in mice and humans in general may be fundamentally different.

\section{Evolutionary Theories in the Light of Genomic Studies}

Clearly, the experiments in which the single gene knock-outs were shown to delay aging in animals are contradictory to the evolutionary theory of aging. Also, some genetic manipulations can delay aging without affecting reproduction [68], which also contradicts the evolutionary theory of aging. Therefore, evolutionary biologists are presented with the task of reconciling these new discoveries, including the single-gene life-extending mutations, with the evolutionary theory of aging.

Nevertheless, the evolutionary theory of aging offers a theoretical framework that explains many observations. The theory offers some indications as to the evolutionary mechanisms and the events leading to the evolution of aging, although it does not offer a complete picture on the evolution of aging across different species. According to Gavrilov and Gavrilova [34], evolutionary theories of aging are useful when they open new opportunities for research by suggesting testable predictions, but they should never be used to impose limitations on aging studies. This is because the evolutionary theories of aging are not in fact complete theories, but rather a set of ideas that themselves require further elaboration and validation [34].

In addition, evolutionary biologists do not expect any single theory to provide all the answers, but rather see the process of aging as involving a combination of theoretical causes. Consequently, the ongoing research will determine through a systems biology approach the degree to which each theory contributes to the actual process of aging [69].

The emerging view from the genomics experiments is that the aging process is quite different in mice and humans, emphasizing the need for research using human samples to uncover aging mechanisms relevant to human longevity. A small number of age-related changes in expression are conserved across species. These aging pathways may be linked to functions in young adults or may be unavoidable consequences of growing old. Identification of these pathways is crucial because they highlight specific aging pathways that can be dissected in model organisms to elucidate general principles of aging [18].

Development of functional genomics tools has made it possible to define the aging process by performing genome-wide scans for transcriptional differences between the young and the old using gene array technology assessment. Global screens for age regulation have been performed for worms and flies, as well as many tissues in mice and humans. A series of recent works has compared age-related expression profiles in worms, mice, flies and humans. For worms and flies, DNA microarrays have been used on whole animals over the entire lifespan to profile transcriptional changes of aging [70]. Recently, Zahn et al. [67] have used data from AGEMAP (Atlas of Gene Expression in Mouse Aging Project), which is a large database of expression changes as a function of age in 16 mouse tissues. They found great heterogeneity in the amount of transcriptional change with age in different tissues. Some tissues displayed large transcriptional differences in old mice, suggesting that these tissues may contribute strongly to organism decline. Other tissues showed few or no changes in expression with age, indicating strong levels of homeostasis throughout life. Based on the pattern of age related transcriptional changes, they found that tissues could be classified into 1 of 3 aging processes: (1) a pattern common to neural tissues; (2) a pattern for vascular tissues, and (3) a pattern for steroidresponsive tissues. They observed that different tissues age in a coordinated fashion in individual mice, such that certain mice exhibit rapid aging, whereas others exhibit slow aging for multiple tissues [67]. 
Transcriptional profiles for aging contain quantitative data on age-related changes in expression for a large fraction of the genome. Genes that show age-related transcriptional differences in multiple species are exceptionally interesting as biomarkers for age. Their age-related decline scales with lifespan, such that age-related changes occur relatively quickly in short-lived animals but slowly in long-lived ones. By contrast, genes that show age regulation in mice but not humans may help identify pathways and mechanisms that account for the much longer lifespan of humans.

\section{Challenges for Future Research in Biogerontology}

Modern biogerontology is characterized by several major and important discoveries which include:

(1) Lifespan extension (in rodents) by caloric restriction, provided that protein, vitamins and micronutrients are sufficiently available to prevent malnutrition.

(2) The occurrence of stress-resistance of long-lived mutant worm strains.

(3) The existence of single gene mutants which extend mouse longevity.

(4) The role of insulin and insulin-like signals in modulating aging rates in widely separated species and animals.

These experimental findings have paved the way for evolutionary approaches to make a substantial contribution to the biology of aging and, ultimately, to medicine [71]. For example, Partridge and Gems [71] have recently given emphasis to the fact that a largely parallel body of work in biogerontology has produced descriptions of the phenotypes of aging and experimental analysis of their mechanistic basis. There have also been some interactions between these 2 approaches: for example, the phenomenon of cellular senescence, which can result in the presence of useless or even damaging cells in the dividing tissues of older humans, can be understood as a sideeffect of a mechanism for preventing cancer [72]. Similarly, several of the neurodegenerative diseases of aging, such as Huntington's and Alzheimer's, might arise as a result of the inadequacy of energetically expensive cellular defense mechanisms [73]. Also, work on progeria, or Hutchinson-Gilford Progeria Syndrome which is caused by a mutation in the LMNA gene on chromosome 1 as well as Werner's syndrome, in which patients have inherited 2 copies of the null allele at the WRN locus on chromosome 8 suggest that the occurrence of age-associated changes can happen as a result of mutation in a sin- gle gene. Because these diseases display different aspects of aging, but never every aspect, they are often called 'segmental progeroid syndrome', a term introduced by George Martin [74, 75]. Thus, the molecular basis of Hutchinson-Gilford syndrome and the details of mutations of the WRN locus may contribute to our understanding of the coordinated appearance of the changes which are general phenomena of the process of normal aging.

Other areas in biogerontology research that may further contribute to our better understanding of evolution of aging can include research works on control of protein synthesis, validation of biomarkers of aging, understanding the biochemistry of longevity, comparative biology studies and research in the fields of gerontologic pathology. For example, both the rate of synthesis and rate of degradation of most proteins is slower in tissues of old rodents and humans, which extends time for the occurrence of different protein modifications and intramolecular rearrangements that may ultimately lead to creation of atypical protein forms and have pathological consequences. Also, the shortening of telomeres, the specialized nucleoprotein structures at the ends of chromosomes, can cause changes in expression of genes nearest the telomere, known as the telomere position effect. As a result, the telomere position effect can result in the agerelated expression and/or over-expression of genes near a telomere that is dependent on both distance from the telomere and individual chromosomal telomere length. In that way, telomere shortening in mitotic somatic cells may contribute to and cause their senescence, accelerate the senescence of neighboring mitotic and postmitotic cells, and underlie organism aging. Accordingly, it has been suggested that if telomere shortening could be slowed or prevented in dividing cells in vivo, this could lead to an increase in replicative lifespan, which may enable these cells to carry out their normal cellular functions for a longer period of time [76]. Another area of particular interest is stem cells research. The embryonic stem cells can differentiate into various cell types. Stem cells from adult tissues also maintain such a potential, if transplanted into the target organ, or cultured in the presence of relevant cytokines and growth factors. In addition, studies on centenarians revealed that their peripheral blood also contains circulating stem cells, albeit at a lower proportion compared to the young adults [77]. However, one of the issues to be solved is the limited capacity of the stem cells from mature tissues to replicate sequentially, as in most of the normal cell types [78]. The aging of tissue-specific stem cell and progenitor cell com- 
partments is believed to be central to the decline of tissue and organ integrity and function in the elderly, and the question of whether or not stem cell aging is accompanied by increased cellular senescence remains an important unresolved issue [79]. Finally, we propose further focus on research elucidating the biochemical and molecular basis for longevity in very old people because data from studies on human longevity showed that nonagenarians and centenarians were healthier than originally predicted on the basis of classical studies on human aging. It thus led to research on the genetics of the healthy very old people in which, for example, it was demonstrated that subjects with exceptional longevity and their offspring have significantly greater high-density lipoprotein levels and particle sizes and lower levels of low-density lipoprotein, which reflect their general health and cognitive function performance [80]. Also, there was the finding of a markedly higher frequency of a functional cholesteryl ester transfer protein (CETP) variant (homozygosity for the 405 valine) in these people, which is the first example of a phenotype and an associated genotype in humans with exceptional longevity [80]. In another study, gender-specific and functionally significant insulin-like growth factor I receptor mutations were described in female centenarians [81]. Moreover, an additional advantageous finding that the CETP-VV genotype was protective in neutralizing the deleterious effects of the lipoprotein(a) gene [82] leads to the proposal that, in fact, long-lived people can have just as many deleterious aging genotypes as the rest of the population and that their protective longevity genotype ('buffering genes' hypothesis) would protect them from the harmful effects of the others [82]. Thus, studies of genotypic frequencies among different age groups can elucidate the genetic determinants and pathways responsible for longevity.

\section{Acknowledgments}

This report is supported by the Krol Foundation of Barnegat, N.J., USA, and a grant from the Technion Vice President for Research.

\section{References}

1 Weismann A: Essays Upon Heredity. Oxford, Clarendon Press, 1891

2 Weismann A: Über die Dauer des Lebens. Fisher, Jena, 1882.

-3 Dyke B, Gage T, Alford P, Swenson B, Williams-Blangero S: Model life table for captive chimpanzees. Am J Primatol 1995;37:25-37.

4 Hill K, Boesch C, Goodall J, Pusey A, Williams J, Wrangham R: Mortality rates among wild chimpanzees. J Hum Evol 2001;40:437450.

5 Austad SN: Retarded senescence in an insular population of opossums (Didelphis virginiana). J Zool 1993;229:695-708.

6 Weismann A: Über Leben und Tod. Fisher, Jena, 1892.

7 Hayflick L, Moorhead PS: The serial cultivation of human diploid cell strains. Exp Cell Res 1961;25:585-621.

8 Hayflick L: The limited in vitro lifetime of human diploid cell strains. Exp Cell Res 1965;37:614-636.

-9 Kerr JF, Wyllie AH, Currie AR: Apoptosis: a basic biological phenomenon with wideranging implications in tissue kinetics. Br J Cancer 1972;26:239-257.

10 Vicencio JM, Galluzzi L, Tajeddine N, Ortiz C, Criollo A, Tasdemir E, Morselli E, Ben Younes A, Maiuri MC, Lavandero S, Kroemer G: Senescence, apoptosis or autophagy? When a damaged cell must decide its path: a mini-review. Gerontology 2008;54:92-99.
11 Weismann A: Essays Upon Heredity and Kindred Biological Problems. Oxford, Clarendon Press, 1889.

12 Kirkwood TB: Evolution of ageing. Nature 1977;270:301-304.

13 Medawar PB: An Unsolved Problem of Biol ogy. London, HK Lewis, 1952.

14 Charlesworth B: Fisher, Medawar, Hamilton and the evolution of aging. Genetics 2000; 156:927-931.

15 Charlesworth B: Evolution in Age-Structured Populations. Cambridge, Cambridge University Press, 1994.

16 Gavrilova NS, Gavrilov LA, Evdokushkina GN, Semyonova VG, Gavrilova AL, Evdokushkina NN, Kushnareva YE, Kroutko VN, Andreyev AY: Evolution, mutation, and human longevity: European royal and noble families. Hum Biol 1998;70:799-804.

17 Strehler BL: Origin and comparison of the effects of time and high energy radiations on living systems. Quart Rev Biol 1959;34:117142.

18 Kim SA: Common aging pathways in worms, flies, mice and humans. J Exp Biol 2007;210: 1607-1612.

19 Carey JR, Liedo P, Orozco D, Vaupel JW: Slowing of mortality-rates at older ages in large medfly cohorts. Science 1992;258:457461.
20 Curtsinger JW, Fukui HH, Townsend DR, Vaupel JW: Demography of genotypes: failure of the limited life-span paradigm in Drosophila melanogaster. Science 1992;258:461463.

21 Charlesworth B: Patterns of age-specific means and genetic variances of mortality rates predicted by the mutation-accumulation theory of ageing. J Theor Biol 2001;210: 47-65.

22 Reynolds RM, Temiyasathit S, Reedy MM, Ruedi EA, Drnevich JM, Leips J, Hughes KA: Age specificity of inbreeding load in Drosophila melanogaster and implications for the evolution of late-life mortality plateaus. Genetics 2007;177:587-595.

23 Williams GC: Pleiotropy, natural selection, and the evolution of senescence. Evolution 1957;11:398-411.

24 Zahavi A: Mate selection: a selection for a handicap. J Theor Biol 1975;53:205-214.

25 Krtolica A, Parrinello S, Lockett S, Desprez PY, Campisi J: Senescent fibroblasts promote epithelial cell growth and tumorigenesis: a link between cancer and aging. Proc Natl Acad Sci USA 2001;98:12072-12077.

26 Cutler RG, Semsei I: Development, cancer and aging: possible common mechanisms of action and regulation. J Gerontol 1989;44: 25-34. 
-27 Economos AC, Lints FA: Developmental temperature and life-span in Drosophilamelanogaster. 1. Constant developmental temperature: evidence for physiological adaptation in a wide temperature-range. Gerontology 1986;32:18-27.

-28 Johnson TE, Hutchinson EW: Absence of strong heterosis for life span and other life history traits in Caenorhabditis elegans. Genetics 1993;134:465-474.

29 Walker DW, McColl G, Jenkins NL, Harris J, Lithgow GJ: Evolution of lifespan in C. elegans. Nature 2000;405:296-297.

-30 Wick G, Berger P, Jansen-Dürr P, GrubeckLoebenstein B: A Darwinian-evolutionary concept of age-related diseases. Exp Gerontol 2003;38:13-25.

-31 Martin GM: Modalities of gene action predicted by the classical evolutionary biological theory of aging. Ann NY Acad Sci 2007; 1100:14-20.

-32 Mannucci PM, Mari D, Merati G, Peyvandi F, Tagliabue L, Sacchi E, Taioli E, Sansoni P, Bertolini S, Franceschi C: Gene polymorphisms predicting high plasma levels of coagulation and fibrinolysis proteins: a study in centenarians. Arterioscler Thromb Vasc Biol 1997; 17:755-759.

$\checkmark 33$ Le Bourg É: A mini-review of the evolutionary theories of aging: is it the time to accept them? Demogr Res 2001;4:1-28.

-34 Gavrilov LA, Gavrilova NS: Evolutionary theories of aging and longevity. Scientific World Journal 2002;2:339-356.

-35 Kirkwood TB, Holliday R: The evolution of ageing and longevity. Proc R Soc Lond B Biol Sci 1979;205:531-546.

-36 Kirkwood TB, Austad SN: Why do we age? Nature 2000;408:233-238.

-37 Congdon JD, Nagle RD, Kinney OM, van Loben Sels RC, Quinter T, Tinkle DW: Testing hypotheses of aging in long-lived painted turtles (Chrysemys picta). Exp Gerontol 2003;38:765-772.

$>38$ Hamilton WD: The moulding of senescence by natural selection. J Theor Biol 1966;12: $12-45$.

>39 Kenyon C, Chang J, Gensch E, Rudner A, Tabtiang RA: C. elegans mutant that lives twice as long as wild type. Nature 1993;366: 461-464.

-40 Kirkwood TB: Evolution of ageing. Mech Ageing Dev 2002;123:737-745.

-41 Gems D, Partridge L: Insulin/IGF signalling and ageing: seeing the bigger picture. Cur Opin Genet Dev 2001;11:287-292.

42 Finch CE, Ruvkun G: The genetics of aging. Annu Rev Genomics Hum Genet 2001;2: 435-462.

43 Kenyon C: The plasticity of aging: insights from long-lived mutants. Cell 2005;120: 449-460.

44 Johnson T: Increased life-span of age-1 mutants in Caenorhabditis elegans and lower Gompertz rate of aging. Science 1990;249: 908-912.
45 Hertweck M, Gobel C, Baumeister R: C. elegans SGK-1 is the critical component in the Akt/PKB kinase complex to control stress response and life span. Dev Cell 2004;6:577588

46 Ogg S, Paradis S, Gottlieb S, Patterson GI, Lee L, Tissenbaum HA, Ruvkun G: The Forkhead transcription factor DAF-16 transduces insulin-like metabolic and longevity signals in C. elegans. Nature 1997;389:994-999.

47 Selman C, Lingard S, Choudhury AI, Batterham RL, Claret M, Clements M, Ramadani F, Okkenhaug K, Schuster E, Blanc E, Piper MD, Al-Qassab H, Speakman JR, Carmignac D, Robinson IC, Thornton JM, Gems D, Partridge L, Withers DJ: Evidence for lifespan extension and delayed age-related biomarkers in insulin receptor substrate 1 null mice. FASEB J 2008;22:807-818.

-48 Adler AS, Sinha S, Kawahara TL, Zhang JY, Segal E, Chang HY: Motif module map reveals enforcement of aging by continual NF$\kappa \mathrm{B}$ activity. Genes Dev 2007;21:3244-3257.

49 Adler AS, Kawahara TL, Segal E, Chang HY: Reversal of aging by $\mathrm{NF \kappa B}$ blockade. Cell Cycle 2008;7:556-559.

50 Cai D, Frantz JD, Tawa Jr NE, Melendez PA, Oh BC, Lidov HG, Hasselgren PO, Frontera WR, Lee J, Glass DJ, Shoelson SE: IKK $\beta / N F-$ $\kappa \mathrm{B}$ activation causes severe muscle wasting in mice. Cell 2004;119:285-298.

51 Arkan MC, Hevener AL, Greten FR, Maeda S, Li ZW, Long JM, Wynshaw-Boris A, Poli G, Olefsky J, Karin M: IKK- $\beta$ links inflammation to obesity-induced insulin resistance. Nat Med 2005;11:191-198.

>52 Chen J, Zhou Y, Mueller-Steiner S, Chen LF, Kwon H, Yi S, Mucke L, Gan L: SIRT1 protects against microglia-dependent amyloid$\beta$ toxicity through inhibiting NF- $\kappa \mathrm{B}$ signaling. J Biol Chem 2005;280:40364-40374.

53 Bar-Shai M, Reznick AZ: Peroxynitrite induces an alternative NF-kappaB activation pathway in L8 rat myoblasts. Antioxid Redox Signal 2006;8:639-652.

54 Bar-Shai M, Carmeli M, Coleman E, Rozen R, Perek N, Fuchs S, Reznick AZ: The effect of hindlimb immobilization on acid phosphatase, metalloproteinases and nuclear factor-kappaB in muscles of young and old rats. Mech Ageing Dev 2005;126:289-297.

55 Bar-Shai M, Carmeli E, Ljubuncic P, Reznick AZ: Exercise and immobilization in aging animals: the involvement of oxidative stress and NFkB activation. Free Radic Biol Med 2007;44:202-214

56 Reed T, Dick DM, Uniacke SK, Foroud T, Nichols WC: Genome-wide scan for a healthy aging phenotype provides support for a locus near D4S1564 promoting healthy aging. J Gerontol A Biol Sci Med Sci 2004;59:227232.

57 Sengupta N, Seto E: Regulation of histone deacetylase activities. J Cell Biochem 2004; 93:57-67.
58 Lin SJ, Ford E, Haigis M, Liszt G, Guarente $\mathrm{L}$ : Calorie restriction extends yeast life span by lowering the level of NADH. Genes Dev 2004; 18:12-16.

-59 Tissenbaum HA, Guarente L: Increased dosage of a sir-2 gene extends lifespan in Caenorhabditis elegans. Nature 2001;410:227230.

60 Rogina B, Helfand SL: Sir2 mediates longevity in the fly through a pathway related to calorie restriction. Proc Natl Acad Sci USA 2004;101:15998-16003.

61 Morrow G, Tanguay RM: Heat shock proteins and aging in Drosophila melanogaster. Semin Cell Develop Biol 2003;14:291-299.

62 Yokoyama K: Extended longevity of Caenorhabditis elegans by knocking in extra copies of hsp70F, a homolog of mot-2 (mortalin)/mthsp70/Grp75. FEBS Lett 2002;516: 53-57.

63 Hsu AL, Murphy CT, Kenyon C: Regulation of aging and age-related disease by DAF-16 and heat-shock factor. Science 2003;300: 1142-1145.

64 Longo VD: Search for methuselah genes heats up. Sci Aging Knowledge Environ 2004;6:pe6

65 Zahn JM, Sonu R, Vogel H, Crane E, MazanMamczarz K, Rabkin R, Davis RW, Becker KG, Owen AB, Kim SK: Transcriptional profiling of aging in human muscle reveals a common aging signature. PLoS Genet 2006; 2:e115.

66 Golden TR, Hinerfeld DA, Melov S: Oxidative stress and aging: beyond correlation. Aging Cell 2002;1:117-123.

67 Zahn JM, Poosala S, Owen AB, Ingram DK, Lustig A, Carter A, Weeraratna AT, Taub DD, Gorospe M, Mazan-Mamczarz K, Lakatta EG, Boheler KR, Xu X, Mattson MP, Falco G, Ko MSH, Schlessinger D, Firman J, Kummerfeld SK, Wood WH 3rd, Zonderman AB, Kim SK, Becke KG: AGEMAP: A Gene Expression Database for Aging in Mice. PLoS Genet 2007;3:e201.

68 Simon AF, Shih C, Mack A, Benzer S: Steroid control of longevity in Drosophila melanogaster. Science 2003;299:1407-1410.

69 Kirkwood TB: Understanding ageing from an evolutionary perspective. J Intern Med 2008;263:117-127.

70 Lund J, Tedesco P, Duke K, Wang J, Kim SK, Johnson TE: Transcriptional profile of aging in C. elegans. Curr Biol 2002;12:1566-1573.

71 Partridge L, Gems D: Beyond the evolutionary theory of ageing, from functional genomics to evo-gero. Trends Ecol Evol 2006; 21:334-340.

72 Campisi J: Senescent cells, tumor suppression, and organismal aging: good citizens, bad neighbors. Cell 2005;120:513-522.

73 Driscoll M, Gerstbrein B: Dying for a cause: invertebrate genetics takes on human neurodegeneration. Nat Rev Genet 2003;4:181194 
74 Ruvalcaba RH, Churesigaew S, Myhre SA, Kelley VC, Martin GM: Children who age rapidly: progeroid syndromes: case report of a new variant. Clin Pediatr (Phila) 1997;16: 248-252.

75 Martin GM, Oshima J: Lessons from human progeroid syndromes. Nature 2000;408: 263-266.

76 Campisi J: Cancer, aging and cellular senescence. In Vivo 2000;14:183-188.
77 Bagnara GP, Bonsi L, Strippoli P, Bonifazi F, Tonelli R, D'Addato S, Paganelli R, Scala E, Fagiolo U, Monti D, Cossarizza A, Bonafé M, Franceschi C: Hemopoiesis in healthy old people and centenarians: well-maintained responsiveness of CD34+ cells to hemopoietic growth factors and remodeling of cytokine network. J Gerontol A Biol Sci Med Sci 2000;55:B61-B66; commentary B69-B70.

78 Sharpless NE, Depinho RA: How stem sells age and why this makes us grow old. Nat Rev Mol Cell Biol 2007;8:703-713.

-79 Rosi DJ, Jamieson CH, Weissman IL: Stems cells and the pathways to aging and cancer. Cell 2008;132:681-696.
80 Atzmon G, Rincon M, Rabizadeh P, Barzilai $\mathrm{N}$ : Biological evidence for inheritance of exceptional longevity. Mech Ageing Dev 2005; 126:341-345.

81 Suh Y, Atzmon G, Cho MO, Hwang D, Liu B, Leahy DJ, Barzilai N, Cohen P: Functionally significant insulin-like growth factor I receptor mutations in centenarians. Proc Natl Acad Sci USA 2008;105:3438-3442.

82 Bergman A, Atzmon G, Ye K, MacCarthy T, Barzilai N: Buffering mechanisms in aging: a systems approach toward uncovering the genetic component of aging. PLoS Comput Biol 2007;3:e170. 